\title{
Differentially-expressed genes in rice infected by Xanthomonas oryzae pv. oryzae relative to a flagellin-deficient mutant reveal potential functions of flagellin in host-pathogen interactions
}

\author{
Chao $\mathrm{Yu}^{1+}$, Huamin $\mathrm{Chen}^{1+}$, Fang Tian ${ }^{1}$, Jan E Leach ${ }^{2}$ and Chenyang $\mathrm{He}^{1 *}$
}

\begin{abstract}
Background: Plants have evolved a sensitive defense response system that detects and recognizes various pathogen-associated molecular patterns (PAMPs) (e.g. flagellin) and induces immune responses to protect against invasion. Transcriptional responses in rice to PAMPs produced by Xanthomonas oryzae pv. oryzae (Xoo), the bacterial blight pathogen, have not yet been defined.

Results: We characterized transcriptomic responses in rice inoculated with the wildtype (WT) Xoo and flagellin-deficient mutant $\Delta$ fliC through RNA-seq analysis. Digital gene expression (DGE) analysis based on Solexa/lllumina sequencing was used to investigate transcriptomic responses in 30 day-old seedlings of rice (Oryza sativa L. cv. Nipponbare). 1,680 genes were differentially-expressed (DEGs) in rice inoculated with WT relative to $\triangle$ flic; among which 1,159 genes were upregulated and 521 were down-regulated. Expression patterns of 12 randomly-selected DEGs assayed by quantitative real time PCR (qRT-PCR) were similar to those detected by DGE analyses, confirming reliability of the DGE data. Functional annotations revealed the up-regulated DEGs are involved in the cell wall, lipid and secondary metabolism, defense response and hormone signaling, whereas the down-regulated ones are associated with photosynthesis. Moreover, 57 and 21 specifically expressed genes were found after WT and $\Delta$ flic treatments, respectively.

Conclusions: DEGs were identified in rice inoculated with WT Xoo relative to $\triangle$ fliC. These genes were predicted to function in multiple biological processes, including the defense response and photosynthesis in rice. This study provided additional insights into molecular basis of rice response to bacterial infection and revealed potential functions of bacterial flagellin in the rice-Xoo interactions.
\end{abstract}

Keywords: Rice; Differentially-expressed genes (DEGs); Flagellin; Immune response; Xanthomonas oryzae pv. oryzae

\section{Background}

Plants, unlike mammals, do not have an adaptive immunity mechanism, and largely rely on the innate immune system to resist pathogen infection (Jones and Dangl 2006). Pattern-triggered immunity (PTI) and effector-triggered immunity (ETI) are two layers of innate immune system

\footnotetext{
* Correspondence: cyhe@caas.net.cn

${ }^{\dagger}$ Equal contributors

'State Key Laboratory for Biology of Plant Diseases and Insect Pests, Institute of Plant Protection, Chinese Academy of Agricultural Sciences, Beijing 100193, China

Full list of author information is available at the end of the article
}

in plant (Chisholm et al. 2006; Tsuda and Katagiri 2010). Pathogen-associated molecular patterns (PAMPs) are conserved and indispensable molecules, which include bacterial flagellin, elongation factor-Tu (EF-Tu), fungal glucans, chitin, etc. (Zhang and Zhou 2010). PTI is activated through recognition of PAMPs by pattern recognition receptors (PRRs) located on the plant cell surface; the resulting basal defense response prevents potential pathogens from colonizing on plant tissues (Chakravarthy et al. 2009; Chujo et al. 2013). PTI is characterized by induction of reactive oxygen species (ROS), ion fluxes, callose deposition 
in the cell wall and other innate immune responses (Tsuda et al. 2008; Boller and Felix 2009). During the evolution of plant-pathogen interactions, some pathogens acquired the ability to suppress PTI by delivering effectors to plant cells, thus causing disease ( $\mathrm{Li}$ et al. 2005; Chisholm et al. 2006). Then plants developed resistance ( $R$ ) proteins to recognize effectors, leading to activation of effectortriggered immunity (ETI) (Jones and Dangl 2006). Pathogenesis related proteins (PRs) and WRKY transcription factors (WRKY TFs) are two kinds of important proteins involved in defense to pathogens in both PTI and ETI (Chujo et al. 2013).

Flagellin, the main component of a bacterial flagellum, was first identified as an elicitor that induces defense responses in tomato and Arabidopsis thaliana (Felix et al. 1999). Flg22, a 22 amino acid, highly conserved domain within the $\mathrm{N}$ terminus of flagellin, caused these defense responses and a strong inhibition of growth in A. thaliana seedlings (Gomez-Gomez et al. 1999). FLAGELLIN SENSITIVE2 (FLS2), the receptor that recognizes flagellin in A. thaliana, shares structural and functional homologies with known plant resistance proteins (Gomez-Gomez and Boller 2000). Flagellin and flg22 are recognized and bound to the extracelluar domain of FLS2. This induces FLS2 heteromerization with BRASSINOSTEROID INSENSITIVE 1-associate kinase 1 (BAK1) and activates signaling cascades that finally culminate in the induction of defense responses (Heese et al. 2007; Ali and Reddy 2008; Sun et al. 2013). Therefore, FLS2 determined the specificity of flagellin perception in A. thaliana (Chinchilla et al. 2006). OsFLS2, the ortholog of FLS2 in rice, also mediates immune responses induced by flagellin (Takai et al. 2008).

Different responses of rice cells to flagellins from compatible or incompatible bacterial strains have been observed. For example, flagellin purified from an incompatible strain of Acidovorax avenae (N1141), induced the rapid generation of $\mathrm{H}_{2} \mathrm{O}_{2}$ accompanying hypersensitive cell death and the expression of $P A L$, Cht-1, and PBZ1 in cultured rice cells, whereas the flagellin from the compatible strain K1 did not (Che et al. 2000; Tanaka et al. 2003). Introduction of N1141 flagellin gene into rice also trigged immune responses (Takakura et al. 2008). However, flg22 did not induce defense responses in rice, suggesting that the recognition mechanism for flagellin might be different between rice and dicotyledonous plants, such as Arabidopsis and tomato (Felix et al. 1999; Takai et al. 2007). Interestingly, when expressed and purified from Escherichia coli, both types of flagellins induced $\mathrm{H}_{2} \mathrm{O}_{2}$ generation in rice. In addition, a deglycosylated flagellin from the compatible strain induced the same immune responses as the flagellin of incompatible strain did (Hirai et al. 2011). These studies suggested that post-translational modifications of flagellins might be associated with the specific induction of immune responses in rice. However, the molecular mechanism of different immune responses of induced by different flagellins remains largely unknown.

Xanthomonas oryzae pv. oryzae (Xoo), causes bacterial blight disease and can result in serious yield loss in rice production. The Xoo-rice interaction has been studied as a model system to understand the molecular mechanisms of disease resistance responses in monocotyledonous plants (Song et al. 1995; Ronald 1997; Martin et al. 2003). Microarray studies revealed that several signaling components, membrane bound receptor kinases and disease-resistant proteins were significantly induced after Xoo inoculation (Narsai et al. 2013). However, the function of flagellin in Xoo-rice interaction has never been studied. In a previous study, we generated a mutant of Xoo that was deleted in the flagellin gene $\mathrm{fliC}(\Delta f l i C)$; the $\Delta f l i C$ mutant was not motile, and caused more disease (increased lesion lengths) in rice leaves relative to the wildtype (WT) strain (Tian et al. 2014), suggesting that differential rice responses to the WT and $\triangle f l i C$ inoculations exist.

In this study, digital gene expression (DGE) based on Solexa/Illumina sequencing, was applied to identify differentially-expressed genes (DEGs) in rice inoculated with WT Xoo relative to the $\Delta f l i C$ mutant. We identified 1,680 DEGs involved in cell wall and lipid synthesis, secondary metabolism, photosynthesis, defense response, and hormone signaling pathways.

\section{Results}

\section{DGE sequencing in rice leaves inoculated with WT Xoo or $\Delta$ flic}

For DGE sequencing, RNAs from rice leaves inoculated with the WT or $\Delta f l i C$ mutant were extracted to prepare two cDNA libraries for RNA-seq analysis. After filtering to remove reads containing the adapter and poly-N or low quality reads, 36,187,662 and 38,239,937 clean reads remained in the WT and $\triangle f l i C$ libraries, respectively. The sequencing depth was sufficient for the transcriptome coverage in rice (Table 1).

A total of 30,423,983 (84.07\%) and 32,279,312 (84.41\%) clean reads in the two libraries were mapped to the reference genome of rice using bowtie software and allowing a 2-bp mismatch. The GC contents were 52.53\% and 54.93\%, respectively (Table 1). Over $80 \%$ clean tags per library were mapped to the reference database, showing the DGE data was reliable and sufficient for subsequent bioinformatics analysis of gene expression.

The raw sequencing data obtained in this work have been deposited in NCBI's Sequence Read Archive (SRA) and are accessible through SRA Series accession number PRJNA238154, and the accession numbers of WT and $\triangle f l i C$ libraries are SRR1168425 and SRR1168426, respectively (http://www.ncbi.nlm.nih.gov/bioproject/? term $=$ PRJNA238154). 
Table 1 Summary of sequencing data

\begin{tabular}{|c|c|c|c|c|c|c|}
\hline \multirow[t]{2}{*}{ Sample } & \multirow[t]{2}{*}{ Raw tag } & \multicolumn{2}{|l|}{ Clean tag } & \multirow[t]{2}{*}{ GC (\%) } & \multicolumn{2}{|c|}{ Tags mapped to gene } \\
\hline & & $\mathrm{TN}$ & $\mathrm{TP}(\%)$ & & TN & $\mathrm{TP}(\%)$ \\
\hline$\overline{W T}$ & $40,067,282$ & $38,239,937$ & 95.44 & 54.93 & $32,279,312$ & 84.41 \\
\hline$\Delta f l i C$ & $37,643,149$ & $36,187,662$ & 96.13 & 52.53 & $30,423,983$ & 84.07 \\
\hline
\end{tabular}

TN: total number; TP: total percentage.

\section{Identification of DEGs in rice inoculated with WT Xoo relative to $\Delta$ flic}

To compare differential gene transcription in WT- and $\Delta f l i C$-treated rice, two transcriptome profiles were analyzed. As described in materials and methods, the number of clean reads in each library was normalized to the number of transcripts per kilobase of exon model per million mapped reads (FPKM) to obtain the normalized gene expression level, then the relative expression levels were calculated by taking $\Delta f l i C$ treatment as the control. Among the 1,680 DEGs identified, 1,159 were upregulated and 521 were down-regulated in the WT relative to $\Delta f l i C$ treatments (Figure 1). In addition, 57 and 21 DEGs were specifically expressed in WT and $\Delta f l i C$ libraries, respectively (Figure 1B).

To validate the reliability of DGE sequencing, the expression of 12 randomly-selected DEGs were revealed by quantitative real time PCR (qRT-PCR) assays in another independent inoculation experiment. The results showed all of gene expression patterns were consistent to those from the DGE analysis, indicating the DGE data was reliable (Figure 2).

\section{Functional classifications of DEGs}

A total of 1,758 DEGs between WT and $\Delta f l i C$ treatments were categorized into 49 functional groups using GO classifications (Figure 3). In the biological process category, metabolic process was the most dominant group, followed by cellular process, response to stimulus and biosynthetic process. Additionally, there were many DEGs involved in carbohydrate metabolism, photosynthesis and secondary metabolism. Among the cellular components, cell was the largest group, followed by cell part and cytoplasm. Catalytic activity was the largest group in molecular function, followed by hydrolase activity, and transporter activity. These results indicate that the bacterial flagellin influences expression of genes involved in multiple physiological and biochemical activities in rice.

\section{Metabolism pathway analysis of DEGs}

To further reveal the potential functions of DEGs, an overview of the metabolic processes in rice transcriptionally affected by WT and $\Delta f l i C$ infection was obtained by using MapMan (Thimm et al. 2004). Cell wall, lipids metabolism, secondary metabolism, amino acid synthesis and degradation, photosynthesis, and other metabolic processes were detected (Figure 4). The DEGs associated with photosynthesis showed significant expression differences between WT- and $\Delta$ fliC-treated rice. Among them, genes involved in light reactions, Calvin cycle and ATP synthesis were down-regulated, while those involved in starch and sucrose degradation were up-regulated (Additional file 1: Table S5). These results suggest that bacterial flagellin suppresses photosynthetic assimilation in rice.

As the first barrier to prevent pathogen infection in plant, the cell wall is important in plant-pathogen interactions.
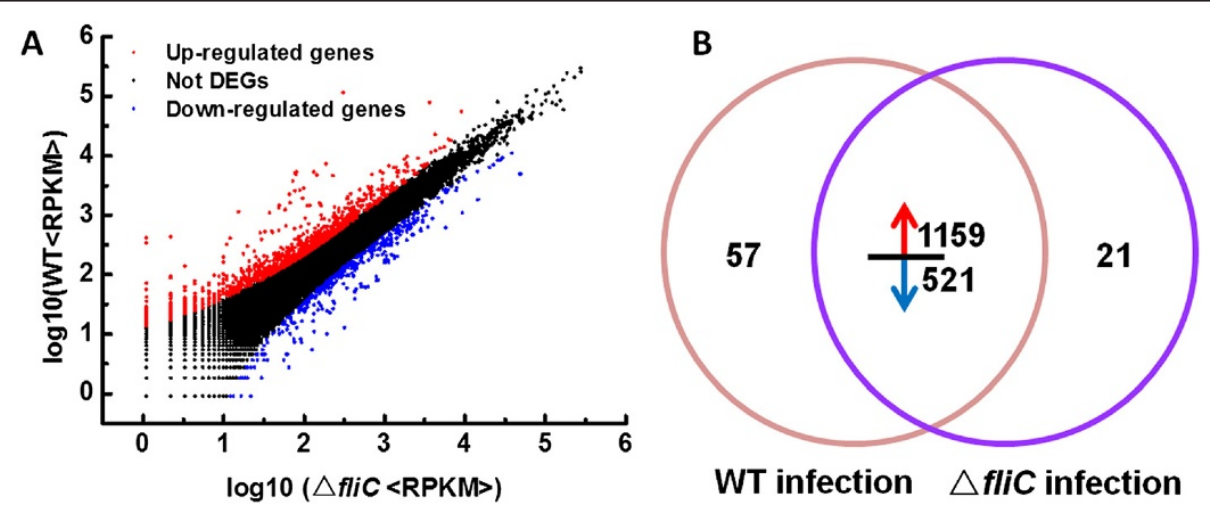

Figure 1 Differentially-expressed genes (DGEs) between the WT and $\Delta$ flic treatments in rice. A, Comparison of gene expression levels between WT and $\Delta f l i C$ treatments. Red and blue dots represent transcripts more prevalent in WT and $\Delta$ flic libraries, respectively. Black dots indicate transcripts that did not change significantly. The parameters " $\mathrm{P} \leq 0.05$ " and " $\log _{2}$ Ratio| $\geq 1$ " were used as the threshold to judge the significance of gene expression difference. B, Changes in gene expression between the WT and $\triangle$ flic libraries. Using the $\triangle$ fliC library as control, red and blue arrows represent genes were up-regulated and down-regulated in WT library, respectively. Data represent the number of DEGs. 


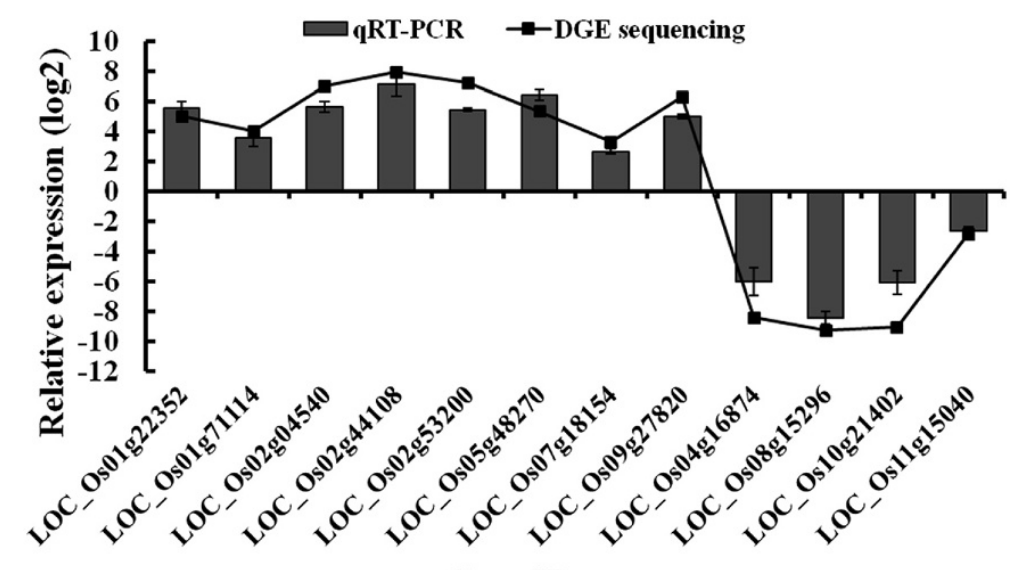

Gene ID

Figure 2 Confirmation of expression of 12 randomly-selected DEGs by qRT-PCR assays. Lines indicate expression from DEG analysis and columns indicate relative expression as measured by qRT-PCR. Bars indicate standard error.

Most of DEGs associated with cell wall structure were upregulated in the WT relative to $\triangle f l i C$-treated rice, including cell wall proteins, cellulose and hemicellulose synthesis and other genes involved in cell wall modification. However, DEGs encoding pectin esterase and pectate lyases were down-regulated (Additional file 2: Table S2).

Lipid metabolism has an important role in defense response to pathogen infection by providing the energy. Five phospholipid synthesis-related DEGs and one glycolipid synthesis-associated DEG were up-regulated in WT relative to $\triangle \mathrm{fliC}$-treated rice. Most of DEGs involved in fatty acid synthesis and degradation were induced, while four DEGs involved in fatty acid desaturation were significantly repressed (Figure 4). These results indicated that lipid metabolism in rice is accelerated by flagellin.

Secondary metabolism is closely linked with defense responses to biotic stresses in plant. In this study, DEGs involved in terpene, flavonoid, phenylpropanoid, phenolic and other metabolism pathways were detected. Seven of 14 DEGs involved in terpene metabolism were down-regulated, while 19 of the 25 DEGs involved in flavonoid metabolism were up-regulated. Similar to DEGs involved in other metabolism pathways, most of the genes involved in phenylpropanoid and phenolic metabolism pathways were up-regulated. One gene involved in wax metabolism was induced. These data suggest that flagellin might enhance defense responses mediated by secondary metabolism in rice.

Other metabolisms such as amino acid synthesis and degradation, nucleotide metabolism and nitrogen metabolism, were also affected. All DEGs involved in amino acid degradation were up-regulated, while DEGs involved in amino acid synthesis showed varied expression patterns. Only one down-regulated DEG involved in nitrogen metabolism, a gene encoding glutamate synthase, was identified.

\section{Identification of DEGs involved in defense and signaling} in rice

To demonstrate the potential roles of bacterial flagellin in induction of defense responses in rice, the DEGs related to defense were particularly scrutinized between the WT and $\triangle f l i C$ treatments. The DEGs included those with known functions in defense, such as TFs, PRs, kinases, peroxidases, etc. (Figure 5, Additional file 3: Table S3). DEGs encoding TFs, including ERF, bZIP, WRKY, MYB and DOF, were identified. Six of 12 DEGs encoding PRs were up-regulated, and the other six were down-regulated. Fifty one DEGs encoding kinase receptors were detected with expression levels influenced by WT and $\Delta f l i C$ treatments. Nine of 10 DEGs encoding peroxidases and seven encoding glutathione-S-transferase were up-regulated.

DEGs involved in signaling of auxin, brassinosteriod, ABA, ethylene, salicylic acid, and jasmonic acid were revealed (Figure 5 and Additional file 4: Table S4). Compared with the $\triangle f l i C$ treatment, five of seven DEGs associated with auxin synthesis, degradation and transport were upregulated by the WT treatment, while two other DEGs were down-regulated. All DEGs involved in brassinosteriod, $\mathrm{ABA}$ and jasmonic acid synthesis and degradation were upregulated. Six of seven DEGs associated with salicylic acid signaling pathways were down-regulated. In addition, 17 of 20 DEGs involved in ethylene signaling pathways were upregulated.

\section{Identification of specifically-expressed genes in WT or $\Delta$ flic treatments}

Fifty-seven specifically expressed genes (SEGs) were found after WT inoculation. The functions of SEGs were associated with the cell wall, secondary metabolism, hormone metabolism, protein degradation and modification, RNA transcription, signaling, development, transport, etc. (Figure 6 and Additional file 5: 


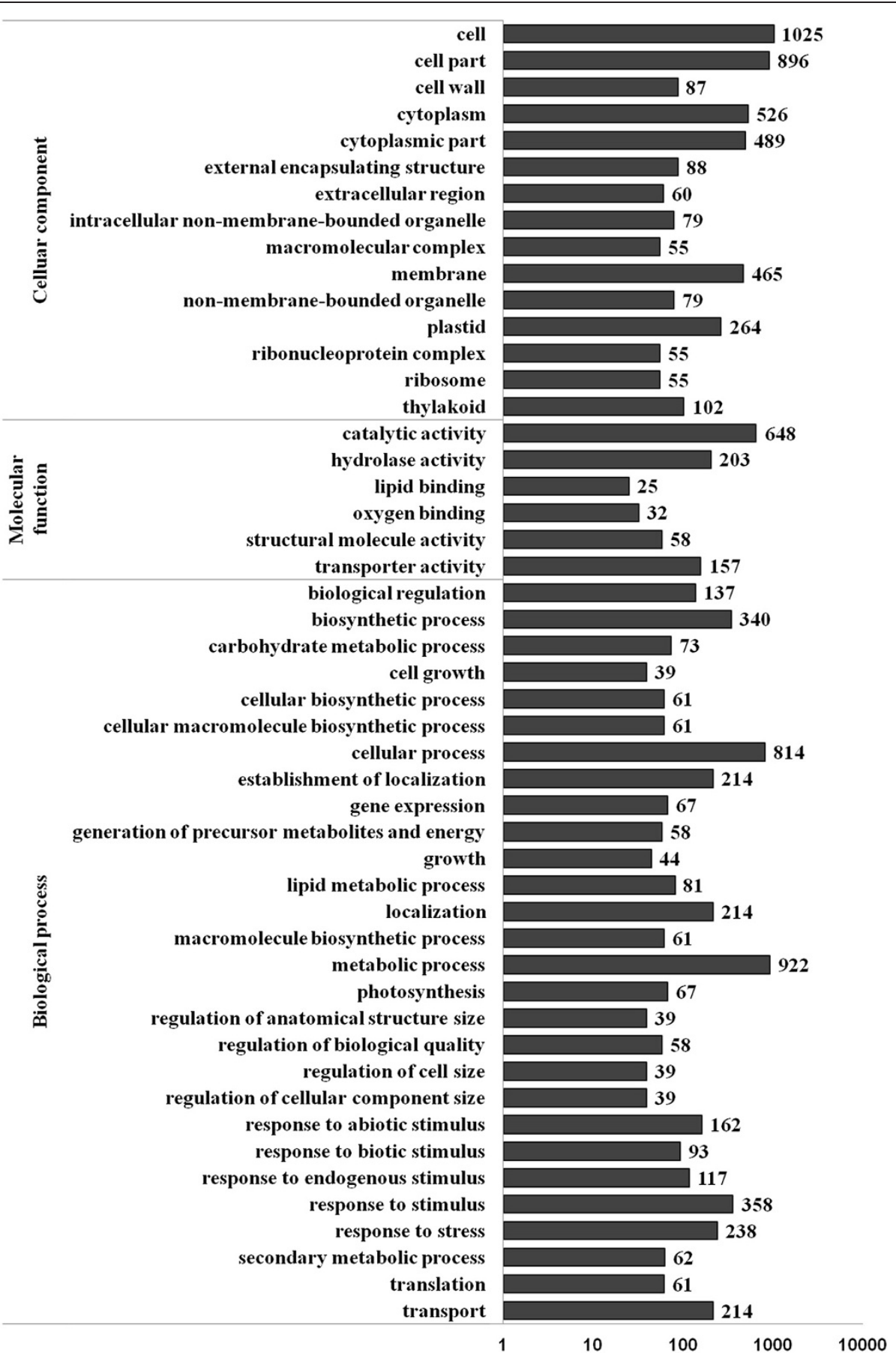

Figure 3 Histogram showing gene ontology functional analysis of DEGs. The $y$-axis and $X$-axis indicated the names of clusters and the number of DEGs in clusters, respectively.

Table S6). In addition, 21 SEGs in the $\Delta f l i C$-treated library were identified to be involved in secondary metabolism, RNA transcription, protein degradation and modification, signaling and transport (Figure 7 and Additional file 6: Table S7).

\section{Discussion}

Flagellin, the main component of flagella, not only plays an important role in bacterial motility, but it is also important in plant PTI (Lee et al. 2003). Flagellin from avirulent but not virulent strains of the rice pathogen 


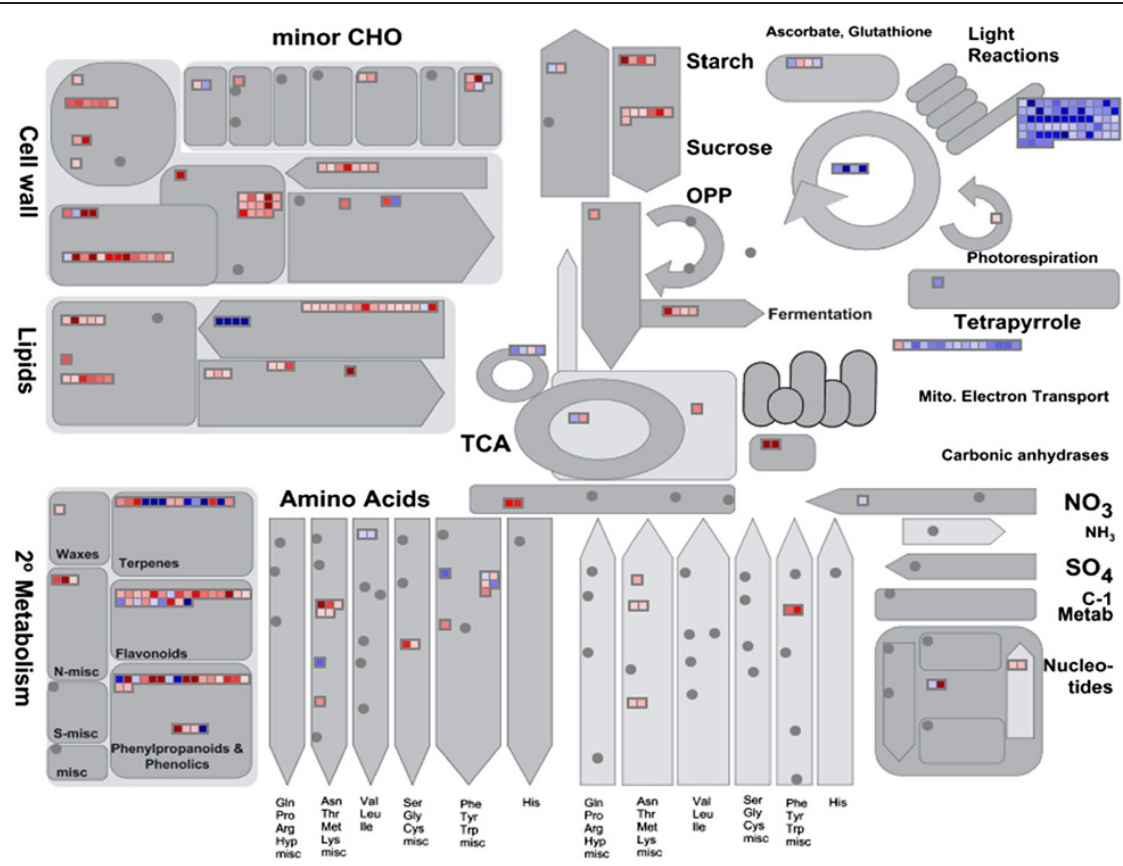

Figure 4 Metabolism overview between WT and $\Delta$ fliC treatments by MapMan. 1,680 DGEs (fold changes $\geq 2, p$-value $\leq 0.05$ ) were imported into MapMan. DGEs up- and down-regulated are represented with red and blue squares, respectively. The grey circles means no differentially-expressed genes involved in this process. Values are $\log _{2}$-transformed fold changes.

Acidovorax avenae induced hypersensitive cell death in cultured rice cells (Che et al. 2000); the specificity of this induction of immune responses was attributed to flagellin glycosylation (Taguchi et al. 2010; Hirai et al. 2011), and suggested that the function of bacterial flagellin in PTI in rice relied on its origin and post-translational modification.
We have shown that a $\mathrm{fliC}$ deletion mutant of Xoo causes increased disease (longer lesion lengths) in rice leaves relative to WT Xoo (Tian et al. 2014). In this study, we identified DEGs by comparing transcriptomic responses in rice inoculated with the WT Xoo and $\triangle \mathrm{fliC}$. The induction of $\mathrm{fliC}$ gene expression of Xoo during bacterial growth in rice

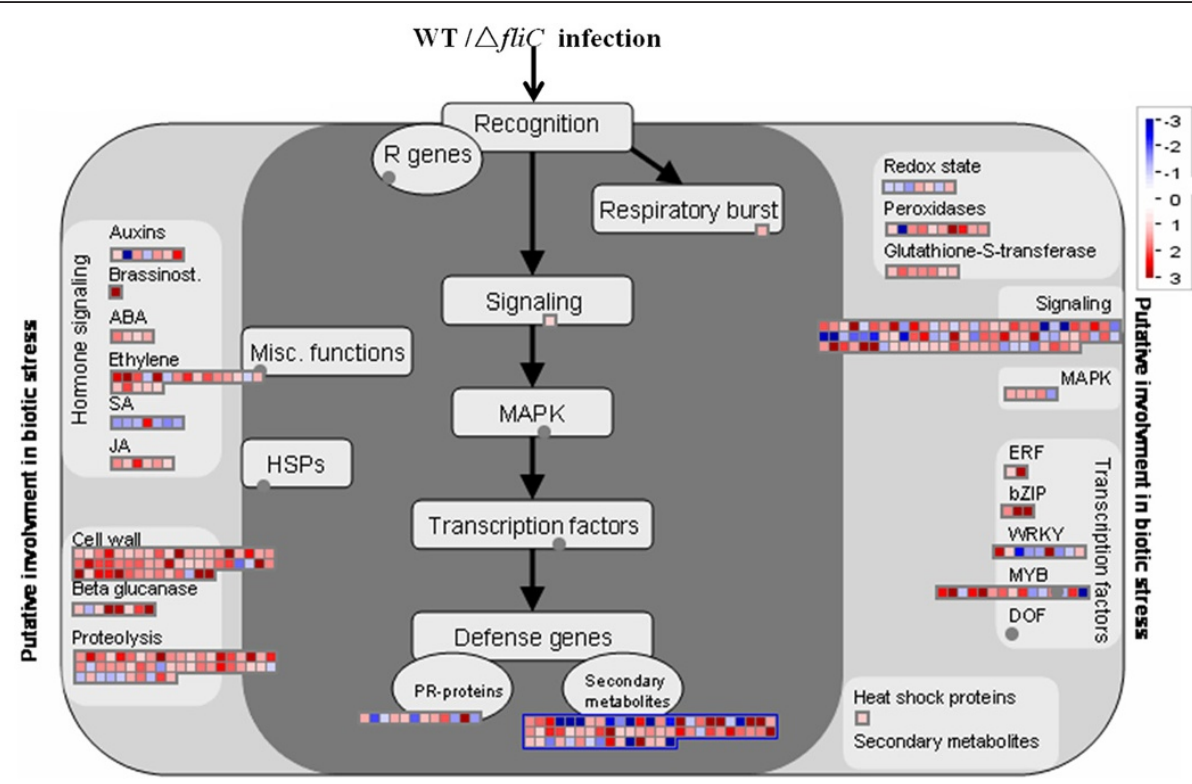

Figure 5 MapMan visualization of defense response between WT and $\Delta$ fliC treatments. 1,680 DGEs (fold changes $\geq 2, p$-value $\leq 0.05$ ) were imported into MapMan. DGEs up- and down-regulated are represented with red and blue squares, respectively. The grey circles means no differentially-expressed genes involved in this process. Values are $\log _{2}$-transformed fold changes. 


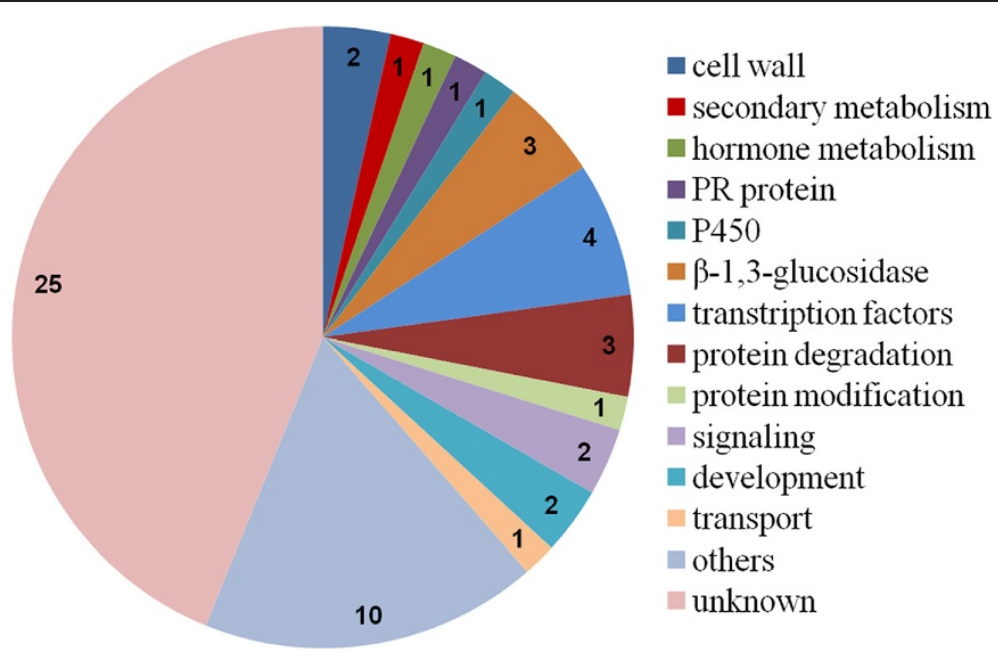

Figure 6 Genes specifically expressed in the WT library. Data represent the number of DEGs.

indicated the expression levels of these DEGs were changed with flagellated Xoo invasion (Additional file 7: Figure S1). The putative functions of these DEGs revealed multiple biological processes, including cell wall synthesis, photosynthesis, defense response and hormone signaling, etc. are involved in rice responses to Xoo flagellin, and provide additional insights into the roles of bacterial flagellin played in the Xoo-rice interactions.

Expression of genes involved in cell wall formation and degradation were influenced by bacterial infection. Microbial pathogens enhance infection by degrading plant cell walls using cell-wall-degrading enzymes. On the other hand, cell wall degradation products produced by microbial activity can induce innate immune responses in plants, including callose deposition and programmed cell death (Jha et al. 2007; Aparna et al. 2009).
In addition, some genes involved in cell wall modification play an important role in basal disease resistance (An et al. 2008). Our current finding that up-regulation of DEGs associated with cell wall precursor synthesis, cell wall degradation and modification (Figure 4 and Additional file 2: Table S2) is consistent with other reports (Narsai et al. 2013; Socquet-Juglard et al. 2013).

PRs are synthesized during basal defense responses and enhanced disease resistance to pathogens in plants. Flagellin induces the expression of PR genes in Arabidopsis (Gomez-Gomez et al. 1999). WRKY TFs form a large family of plant-specific transcription factors and can be positively or negatively regulated during defense responses (Chujo et al. 2013). Constitutive expression of WRKY18 enhanced resistance to Pseudomonas syringae in Arabidopsis, while its co-expression with WRKY40

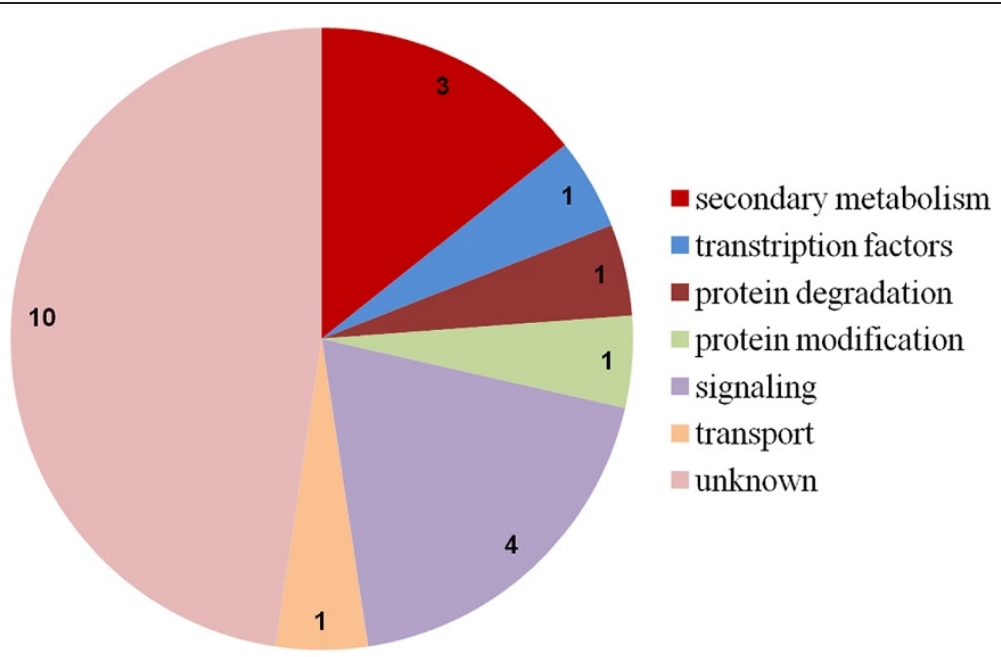

Figure $\mathbf{7}$ Genes specifically expressed in the $\Delta$ fliC library. Data represent the number of DEGs. 
made plants more susceptible to $P$. syringae (Xu et al. 2006; Schon et al. 2013). Our results revealed seven upregulated PRs involved in basal disease resistance, and down-regulated genes for WRKY18 and WRKY40 (Figure 5 and Additional file 3: Table S3), demonstrating these particular genes might play important roles in rice-Xoo interactions.

Pathogen infection usually results in the significant decreases of photosynthesis, and in turn, limits the availability of nutrient sources for pathogen (Berger et al. 2007). For example, the photosynthesis was decreased in Arabidopsis leaves after challenged with P. syringae (Bonfig et al. 2006). Genes encoding photosynthesis components were repressed by Xoo infection in rice (Narsai et al. 2013). Activation of defense by PAMPs leads to rapid decrease in nonphotochemical quenching (Gohre et al. 2012). Our data showed genes encoding proteins involved in light reactions, Calvin cycle and ATP synthesis were repressed in rice after treatment with WT Xoo relative to the mutant (Figure 4 and Additional file 4: Table S4), implying the bacterial flagellin might function in suppression of photosynthesis of rice.

Roles of salicylic acid (SA) and ethylene (ET)/jasmonic acid (JA)-mediated signaling pathways in resistance to pathogens have been well studied (Robert-Seilaniantz et al. 2011). SA synthesis was accelerated by pathogen infection, and enhanced the induction of defense gene transcripts, $\mathrm{H}_{2} \mathrm{O}_{2}$ accumulation and hypersensitive cell death in plants (Gaffney et al. 1993; Shirasu et al. 1997), and ET/JA signaling pathway was required for resistance to necrotrophic pathogens(Glazebrook 2005; Denance et al. 2013). In addition, ET synthesis was induced by the PAMP flg22 (Felix et al. 1999). In our study, several genes encoding 1-aminocyclopropane-1carboxylate oxidase (ACO) and 12-oxophytodienoate reductase (OPR) were up-regulated (Figure 5 and Additional file 1: Table S5); these are key enzymes involved in ET and JA synthesis pathway, respectively (Vriezen et al. 1999; Schaller et al. 2000). These results indicated ET/JA synthesis were induced by Xoo flagellin in rice. Interestingly, seven genes encoding salicylic acid carboxyl methyltransferase (SAMT), catalyzing SA to methyl SA or dimethyl SA (Fukami et al. 2002), were down-regulated. We suggest that SA accumulation in rice is induced by Xoo flagellin. Genes involved in auxin and brassinosteroid signaling pathways were also identified, but the relationship of these hormones and plant defense response is largely unknown.

\section{Conclusion}

A number of rice genes differentially expressed after inoculation with WT Xoo relative to $\triangle f l i C$ was identified by DGE analysis, some of which were validated by qRT-PCR assays for their expression patterns. These DEGs were involved in the multiple different biological functions, including induction of cell wall and lipid synthesis, secondary metabolism, defense responses, hormone signaling and suppression of photosynthesis. This study provided additional insights into molecular basis of rice response to bacterial infection and revealed potential functions of bacterial flagellin in the rice-Xoo interactions. Functional characterization is further required for these candidate genes of rice via the transgenic approaches including the gene over-expression and/or silencing analysis.

\section{Methods}

\section{Plant materials and bacterial inoculation treatment}

Rice seeds (Oryza sativa L. cv. Nipponbare) were germinated for 2 days in water at $37^{\circ} \mathrm{C}$. Individual germinated seeds were planted in a small pots containing vermiculite, the pots were placed in a tray that contained $1 \mathrm{~L}$ Hogland's solution, which was a mixture of $10 \mathrm{mM}$

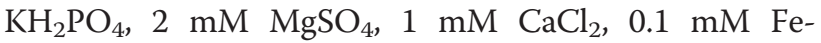
EDTA, $50 \mu \mathrm{M} \mathrm{H} \mathrm{H}_{3} \mathrm{BO}_{4}, 12 \mu \mathrm{M} \mathrm{MnSO}_{4}, 1 \mu \mathrm{M} \mathrm{ZnCl}$, $1 \mu \mathrm{M} \mathrm{CuSO}, 0.2 \mu \mathrm{M} \mathrm{Na}_{2} \mathrm{MoO}_{4}$ and $3 \mathrm{mM} \mathrm{KNO}_{3}$. The $\mathrm{pH}$ of the nutrient solution was adjusted to 5.5-6 using $50 \%$ phosphoric acid, and the solution was replaced every two days. Growth conditions were $16 \mathrm{~h}$ light $\left(29^{\circ} \mathrm{C}\right)$ and $8 \mathrm{~h}$ of dark $\left(23^{\circ} \mathrm{C}\right)$. Xoo strain PXO99 ${ }^{\mathrm{A}}$ (Hopkins et al. 1992) is a virulent strain to Nipponbare. $\triangle f l i C$, which is a fliC deletion mutant derived from PXO99 ${ }^{\mathrm{A}}$, was generated by marker exchange strategy as previous described (Tian et al. 2014). PXO99 ${ }^{\mathrm{A}}$ and $\Delta$ fliC were grown for $72 \mathrm{~h}$ at $28^{\circ} \mathrm{C}$ in M210 media (Yang et al. 2012), then the cells were collected by centrifugation and resuspended in $\mathrm{d}_{2} \mathrm{H}_{2} \mathrm{O}$ at an $\mathrm{OD}_{600}$ of 0.8 (approximately $1.0 \times 10^{8} \mathrm{CFU} / \mathrm{mL}$ ). Thirty day-old seedlings of rice were inoculated with $\mathrm{PXO} 99^{\mathrm{A}}$ and $\triangle \mathrm{fliC}$ by the leafclipping method (Chen et al. 2002). Five centimeters tip of inoculated rice leaves were collected at $12 \mathrm{~h}$ after inoculation, frozen in liquid nitrogen, and stored at $-80^{\circ} \mathrm{C}$.

In order to detect the $\mathrm{fliC}$ expression of during Xoo growth in rice, we collected the rice leaves, which were inoculated with PXO99 ${ }^{\mathrm{A}}$ at $7 \mathrm{~d}$ after inoculation. Then total RNA was extracted using Trizol reagent (Invitrogen, USA), and treated with DNase. The first strand cDNA fragment of $\mathrm{fliC}$ was synthesized using Superscript III reverse transcriptase (Invitrogen, USA). Specific primer sets, fliC-F (5' -CCGAGCGTTTCACTACC CA- 3') and fliC-R (5' -ATCCTTGAACGACAGGCT GAT - 3') were designed based on the sequence of fliC. PCR was performed with one denaturation cycle of $5 \mathrm{~min}$ at $98^{\circ} \mathrm{C}$ and 32 cycles of $30 \mathrm{~s}$ at $95^{\circ} \mathrm{C}, 30 \mathrm{~s}$ at $55^{\circ} \mathrm{C}$, and $30 \mathrm{~s}$ at $72^{\circ} \mathrm{C}$, then $10 \mathrm{~min}$ at $72^{\circ} \mathrm{C}$. Ten microlitres of the PCR product was loaded onto $2 \%$ agarose gel. The PCR was performed without Superscript III reverse transcriptase as a negative control. 


\section{Solexa/lllumina sequencing}

Total RNA was extracted from rice leaves in WT treatment and $\triangle f l i C$ treatment using Trizol reagent (Invitrogen). According to the manufacturer's instruction of Solexa/lllumina sequencing, mRNA were isolated and enriched from $6 \mu \mathrm{g}$ total RNA by using the oligo(dT) magnetic beads, then the first- and second-strand cDNA were synthesized by oligo(dT) primer. cDNA were digested with NlaIII, and the cDNA fragments were ligated with adapter 1, digested with MmeI to produce tags with adaptor 1 . After removing the magnetic beads, Illumina adaptor 2 was ligated to the $3^{\prime}$ ends of cDNA fragments, and a tag library was formed by different adaptors at both ends of fragments. Lastly, the library fragments were amplified by PCR and purified, and then sequenced via Illumina HiSeq 2000.

\section{Identification of differentially-expressed genes}

Raw data from Solexa/Illumina sequencing were cleaned by removing reads containing the adapter or poly- $\mathrm{N}$, orlow quality reads. At the same time, the Q20 content and GC content and sequence duplication level of the clean data were calculated. All downstream analyses were based on the clean data with high quality. All clean tags were mapped to reference sequences using bowtie software and allowing 2-bp mismatch. The number of clean tags for each gene was calculated and then normalized to reads per kilobase of exon model per million mapped reads (FPKM). Differentially expressed genes between two samples were identified by tophat and cufflinks softwares (Trapnell et al. 2009; Roberts et al. 2011), The $P \leq 0.05$ and the absolute value of $\log _{2}$ Ratio $\geq$ 1 were chosen as the threshold to judge the significance of gene expression difference.

\section{Functional annotation of DEGs}

Gene Ontology (GO) (Ashburner et al. 2000) and MapMan (Thimm et al. 2004) were used to analysis biological function of DEGs. The GO enrichment analysis was applied to describe product characteristics and reaction network of DEGs. All DEGs were mapped to GO terms in the database (http://bioinfo.cau.edu.cn/agriGO/). GO terms with corrected $\mathrm{P}$ value less than 0.05 were considered significantly enriched by differential expressed genes. MapMan software was used to visualize the expression change levels of individual genes in diagrams of metabolic pathways. As previously described (Thimm et al. 2004), an Oryza sativa map was developed and uploaded to MapMan, and the change in expression ratio of each gene was calculated as the $\log _{2^{-}}$ fold change to generate the MapMan experimental file.

\section{Quantitative real-time PCR (qRT-PCR) analysis}

Analysis of qRT-PCR were carried out using SYBR Green detection reagents (Quanta Biosciences, USA) in Applied Biosystem's 7500 Sequence Detection System (Applied
Biosystems, USA), and the $20 \mu \mathrm{l}$ PCR reaction contained about 100 ng of cDNA. The reaction mixture was incubated at $95^{\circ} \mathrm{C}$ for $3 \mathrm{~min}$, and then 40 cycles of $95^{\circ} \mathrm{C}$ for $10 \mathrm{~s}, 60^{\circ} \mathrm{C}$ for $30 \mathrm{~s}$, followed by a disassociation stage. Twelve DEGs were randomly selected, and specific primers of these genes were designed with the software Primer Premier 5.0 (PREMIER Biosoft Int., USA) (Additional file 8: Table S1). The rice actin gene was selected as a reference gene in $\mathrm{qRT}-\mathrm{PCR}$. The relative expression ratio was calculated using $2^{-\Delta \Delta C t}$ method (Schmittgen and Livak 2008). All samples were performed in three biological replicates and triplicate PCR, and the error bars show standard error.

\section{Additional files}

Additional file 1: Table S5. DEGs involved in photosynthesis.

Additional file 2: Table S2. DEGs involved in cell wall.

Additional file 3: Table S3. DEGs involved in defense response.

Additional file 4: Table S4. DEGs involved in hormone signaling.

Additional file 5: Table S6. DEGs specially expressed in WT library.

Additional file 6: Table S7. DEGs specially expressed in $\triangle$ fliC library.

Additional file 7: Figure S1. The expression level of fliC was detected during Xoo growth in-rice. WT: fliC expression of PXO99 ${ }^{A}$ during growth in-rice; N: negative control.

Additional file 8: Table S1. Primer sequences used for the validation of DEGs.

\section{Competing interests}

The authors declare that they have no competing interests.

\section{Author contributions}

Conceived and designed the experiments: CY HMC CYH. Performed the experiments: CY HMC. Analyzed the data: CY HMC FT JEL CYH. Contributed reagents/materials/analysis tools: HMC FT. Wrote the paper: CY HMC FT JEL $\mathrm{CYH}$. All authors read and approved the final manuscript.

\section{Acknowledgements}

This work was supported by the grants from the National Basic Research Program (973) (Grant ${ }^{\#}$ 2011CB100700) and National High-Technology Research Program (863) (Grant ${ }^{\#}$ 2012AA101504) to C. He.

\section{Author details}

${ }^{1}$ State Key Laboratory for Biology of Plant Diseases and Insect Pests, Institute of Plant Protection, Chinese Academy of Agricultural Sciences, Beijing 100193, China. ${ }^{2}$ Department of Bioagricultural Sciences and Pest Management, Colorado State University, Ft. Collins, CO 80523-1177, USA.

Received: 27 March 2014 Accepted: 2 August 2014

Published online: 03 September 2014

\section{References}

Ali GS, Reddy A. PAMP-triggered immunity: Early events in the activation of FLAGELLIN SENSITIVE2. Plant Signal Behav. 2008;3:423-6.

An SH, Sohn KH, Choi HW, Hwang IS, Lee SC, Hwang BK. Pepper pectin methylesterase inhibitor protein CaPMEI1 is required for antifungal activity, basal disease resistance and abiotic stress tolerance. Planta. 2008;228:61-78. Aparna G, Chatterjee A, Sonti RV, Sankaranarayanan R. A cell wall-degrading esterase of Xanthomonas oryzae requires a unique substrate recognition module for pathogenesis on rice. Plant Cell. 2009:21:1860-73.

Ashburner M, Ball CA, Blake JA, Botstein D, Butler H, Cherry JM, Davis AP, Dolinski K, Dwight SS, Eppig JT, Harris MA, Hill DP, Issel-Tarver L, Kasarskis A, Lewis S, Matese JC, Richardson JE, Ringwald M, Rubin GM, Sherlock G. Gene 
ontology: tool for the unification of biology. The Gene Ontology Consortium. Nat Genet. 2000;25:25-9.

Berger S, Sinha AK, Roitsch T. Plant physiology meets phytopathology: plant primary metabolism and plant-pathogen interactions. J Exp Bot. 2007;58:4019-26

Boller T, Felix G. A renaissance of elicitors: perception of microbe-associated molecular patterns and danger signals by pattern-recognition receptors. Annu Rev Plant Biol. 2009;60:379-406.

Bonfig KB, Schreiber U, Gabler A, Roitsch T, Berger S. Infection with virulent and avirulent P-syringae strains differentially affects photosynthesis and sink metabolism in Arabidopsis leaves. Planta. 2006;225:1-12.

Chakravarthy S, Velasquez AC, Martin GB. Assay for pathogen-associated molecular pattern (PAMP)-triggered immunity (PTI) in plants. J Vis Exp. 2009 doi.org/10.1016/j.micres.2014.07.002.

Che FS, Nakajima Y, Tanaka N, Iwano M, Yoshida T, Takayama S, Kadota I, Isogai A. Flagellin from an incompatible strain of Pseudomonas avenae induces a resistance response in cultured rice cells. J Biol Chem. 2000;275:32347-56.

Chen $\mathrm{H}$, Wang $\mathrm{S}$, Zhang Q. New gene for bacterial blight resistance in rice located on chromosome 12 identified from minghui 63, an elite restorer line. Phytopathology. 2002;92:750-4.

Chinchilla D, Bauer Z, Regenass M, Boller T, Felix G. The Arabidopsis receptor kinase FLS2 binds flg22 and determines the specificity of flagellin perception. Plant Cell. 2006:18:465-76.

Chisholm ST, Coaker G, Day B, Staskawicz BJ. Host-microbe interactions: shaping the evolution of the plant immune response. Cell. 2006;124:803-14.

Chujo T, Miyamoto K, Shimogawa T, Shimizu T, Otake Y, Yokotani N, Nishizawa Y, Shibuya N, Nojiri H, Yamane H, Minami E, Okada K. OsWRKY28, a PAMPresponsive transrepressor, negatively regulates innate immune responses in rice against rice blast fungus. Plant Mol Biol. 2013;82:23-37.

Denance N, Sanchez-Vallet A, Goffner D, Molina A. Disease resistance or growth: the role of plant hormones in balancing immune responses and fitness costs. Front Plant Sci. 2013:4:155.

Felix G, Duran JD, Volko S, Boller T. Plants have a sensitive perception system for the most conserved domain of bacterial flagellin. Plant J. 1999;18:265-76.

Fukami H, Asakura T, Hirano H, Abe K, Shimomura K, Yamakawa T. Salicylic acid carboxyl methyltransferase induced in hairy root cultures of Atropa belladonna after treatment with exogeneously added salicylic acid. Plant Cell Physiol. 2002;43:1054-8.

Gaffney T, Friedrich L, Vernooij B, Negrotto D, Nye G, Uknes S, Ward E, Kessmann $\mathrm{H}$, Ryals J. Requirement of salicylic Acid for the induction of systemic acquired resistance. Science. 1993;261:754-6.

Glazebrook J. Contrasting mechanisms of defense against biotrophic and necrotrophic pathogens. Annu Rev Phytopathol. 2005;43:205-27.

Gohre V, Jones AM, Sklenar J, Robatzek S, Weber AP. Molecular crosstalk between PAMP-triggered immunity and photosynthesis. Mol Plant Microbe Interact. 2012;25:1083-92

Gomez-Gomez L, Boller T. FLS2: an LRR receptor-like kinase involved in the perception of the bacterial elicitor flagellin in Arabidopsis. Mol Cell. 2000;5:1003-11.

Gomez-Gomez L, Felix G, Boller T. A single locus determines sensitivity to bacterial flagellin in Arabidopsis thaliana. Plant J. 1999;18:277-84.

Heese A, Hann DR, Gimenez-lbanez S, Jones AM, He K, Li J, Schroeder Jl, Peck SC, Rathjen JP. The receptor-like kinase SERK3/BAK1 is a central regulator of innate immunity in plants. Proc Natl Acad Sci U S A. 2007;104:12217-22.

Hirai H, Takai R, Iwano M, Nakai M, Kondo M, Takayama S, Isogai A, Che FS. Glycosylation regulates specific induction of rice immune responses by Acidovorax avenae flagellin. J Biol Chem. 2011;286:25519-30.

Hopkins CM, White FF, Choi SH, Guo A, Leach JE. Identification of a family of avirulence genes from Xanthomonas oryzae pv. oryzae. Mol Plant Microbe Interact. 1992:5:451-9.

Jha G, Rajeshwari R, Sonti RV. Functional interplay between two Xanthomonas oryzae pv. oryzae secretion systems in modulating virulence on rice. Mol Plant Microbe Interact. 2007;20:31-40.

Jones JD, Dangl JL. The plant immune system. Nature. 2006;444:323-9.

Lee MC, Weng SF, Tseng YH. Flagellin gene fliC of Xanthomonas campestris is upregulated by transcription factor Clp. Biochem Biophys Res Commun. 2003;307:647-52.

Li X, Lin H, Zhang W, Zou Y, Zhang J, Tang X, Zhou JM. Flagellin induces innate immunity in nonhost interactions that is suppressed by Pseudomonas syringae effectors. Proc Natl Acad Sci U S A. 2005;102:12990-5.
Martin GB, Bogdanove AJ, Sessa G. Understanding the functions of plant disease resistance proteins. Annu Rev Plant Biol. 2003;54:23-61.

Narsai R, Wang C, Chen J, Wu J, Shou H, Whelan J. Antagonistic, overlapping and distinct responses to biotic stress in rice (Oryza sativa) and interactions with abiotic stress. BMC Genomics. 2013;14:93.

Roberts A, Pimentel H, Trapnell C, Pachter L. Identification of novel transcripts in annotated genomes using RNA-Seq. Bioinformatics. 2011;27:2325-9.

Robert-Seilaniantz A, Grant M, Jones JD. Hormone crosstalk in plant disease and defense: more than just jasmonate-salicylate antagonism. Annu Rev Phytopathol. 2011:49:317-43.

Ronald PC. The molecular basis of disease resistance in rice. Plant Mol Biol. 1997;35:179-86

Schaller F, Biesgen C, Mussig C, Altmann T, Weiler EW. 12-Oxophytodienoate reductase 3 (OPR3) is the isoenzyme involved in jasmonate biosynthesis. Planta. 2000;210:979-84.

Schmittgen TD, Livak KJ. Analyzing real-time PCR data by the comparative $C(T)$ method. Nat Protoc. 2008:3:1101-8.

Schon M, Toller A, Diezel C, Roth C, Westphal L, Wiermer M, Somssich IE. Analyses of wrky 18 wrky 40 plants reveal critical roles of SA/EDS1 signaling and indole-glucosinolate biosynthesis for golovinomyces orontii resistance and a loss-of resistance towards pseudomonas syringae pv. tomato AvrRPS4. Mol Plant Microb In. 2013;26:758-67.

Shirasu K, Nakajima H, Rajasekhar VK, Dixon RA, Lamb C. Salicylic acid potentiates an agonist-dependent gain control that amplifies pathogen signals in the activation of defense mechanisms. Plant Cell. 1997:9:261-70.

Socquet-Juglard D, Kamber T, Pothier JF, Christen D, Gessler C, Duffy B, Patocchi A. Comparative RNA-seq analysis of early-infected peach leaves by the invasive phytopathogen Xanthomonas arboricola pv. pruni. PLoS One. 2013:8:e54196.

Song WY, Wang GL, Chen LL, Kim HS, Pi LY, Holsten T, Gardner J, Wang B, Zhai WX, Zhu LH, Fauquet C, Ronald P. A receptor kinase-like protein encoded by the rice disease resistance gene, Xa21. Science. 1995;270:1804-6.

Sun Y, Li L, Macho AP, Han Z, Hu Z, Zipfel C, Zhou JM, Chai J. Structural basis for flg22-induced activation of the Arabidopsis FLS2-BAK1 immune complex. Science. 2013;342:624-8.

Taguchi F, Yamamoto M, Ohnishi-Kameyama M, Iwaki M, Yoshida M, Ishii T, Konishi T, Ichinose Y. Defects in flagellin glycosylation affect the virulence of Pseudomonas syringae pv. tabaci 6605. Microbiology. 2010;156:72-80.

Takai R, Kaneda T, Isogai A, Takayama S, Che FS. A new method of defense response analysis using a transient expression system in rice protoplasts. Biosci Biotechnol Biochem. 2007;71:590-3.

Takai R, Isogai A, Takayama S, Che FS. Analysis of flagellin perception mediated by flg22 receptor OsFLS2 in rice. Mol Plant Microbe Interact. 2008:21:1635-42

Takakura Y, Che FS, Ishida Y, Tsutsumi F, Kurotani K, Usami S, Isogai A, Imaseki H. Expression of a bacterial flagellin gene triggers plant immune responses and confers disease resistance in transgenic rice plants. Mol Plant Pathol. 2008; $9: 525-9$

Tanaka N, Che FS, Watanabe N, Fujiwara S, Takayama S, Isogai A. Flagellin from an incompatible strain of Acidovorax avenae mediates $\mathrm{H} 2 \mathrm{O} 2$ generation accompanying hypersensitive cell death and expression of PAL, Cht-1, and PBZ1, but not of Lox in rice. Mol Plant Microbe Interact. 2003;16:422-8.

Thimm O, Blasing O, Gibon Y, Nagel A, Meyer S, Kruger P, Selbig J, Müller LA, Rhee SY, Stitt M. MAPMAN: a user-driven tool to display genomics data sets onto diagrams of metabolic pathways and other biological processes. Plant J. 2004;37:914-39.

Tian F, Yu C, Li HY, Wu XL, Li B, Chen HM, Wu M, He C. Alternative sigma factor RpoN2 is required for flagellar motility and full virulence of Xanthomonas oryzae pv. oryzae. Microbiol Res. 2014 (accepted). doi.org/10.1016/j. micres.2014.07.002.

Trapnell C, Pachter L, Salzberg SL. TopHat: discovering splice junctions with RNA-Seq. Bioinformatics. 2009:25:1105-11.

Tsuda K, Katagiri F. Comparing signaling mechanisms engaged in patterntriggered and effector-triggered immunity. Curr Opin Plant Biol. 2010;13:459-65.

Tsuda K, Sato M, Glazebrook J, Cohen JD, Katagiri F. Interplay between MAMPtriggered and SA-mediated defense responses. Plant J. 2008;53:763-75.

Vriezen WH, Hulzink R, Mariani C, Voesenek LA. 1-aminocyclopropane-1-carboxylate oxidase activity limits ethylene biosynthesis in Rumex palustris during submergence. Plant Physiol. 1999:121:189-96. 
Xu XP, Chen CH, Fan BF, Chen ZX. Physical and functional interactions between pathogen-induced Arabidopsis WRKY18, WRKY40, and WRKY60 transcription factors. Plant Cell. 2006;18:1310-26.

Yang F, Tian F, Sun L, Chen H, Wu M, Yang CH, He C. A novel two-component system PdeK/PdeR regulates c-di-GMP turnover and virulence of Xanthomonas oryzae pv. oryzae. Mol Plant Microbe Interact. 2012;25:1361-9.

Zhang J, Zhou JM. Plant immunity triggered by microbial molecular signatures. Mol Plant. 2010;3:783-93.

doi:10.1186/s12284-014-0020-7

Cite this article as: Yu et al.: Differentially-expressed genes in rice infected by Xanthomonas oryzae pv. oryzae relative to a

flagellin-deficient mutant reveal potential functions of flagellin

in host-pathogen interactions. Rice 2014 7:20.

\section{Submit your manuscript to a SpringerOpen ${ }^{\circ}$} journal and benefit from:

- Convenient online submission

- Rigorous peer review

- Immediate publication on acceptance

- Open access: articles freely available online

- High visibility within the field

- Retaining the copyright to your article

Submit your next manuscript at $\gg$ springeropen.com 